Abstract P0-0295 Table 1 Growth data terms vs preterms

\begin{tabular}{|c|c|c|}
\hline & Preterms $<36 \mathrm{w}$ & Full terms $>37$ w \\
\hline Number & $n=60$ & $n=155$ \\
\hline Gestational age & $315+/-2.2$ & $40+-1.5$ \\
\hline Weight (g) & $1584+/-451$ & $3151+/-597 *$ \\
\hline Length (cm) & $41.4+/ 5$ & $50.7+i-3.5 *$ \\
\hline BMI & $9+/-1.1$ & $12.2+/ .2$ * \\
\hline Mo ther wt (kg) & $59.8+16.2$ & $66.3+t-18$ * \\
\hline Head Circumf. (cm) & $28.9+/-2.8$ & $34+1-2 *$ \\
\hline Total Cakium & $2.11+/ .0 .2$ & $231+/-0.16$ \\
\hline Hyp ocalcemia \% & $3 \%$ & $3 \%$ \\
\hline Hemoglobin (g/d) & $16.4+1-25$ & $15.7+/ 2.45$ \\
\hline Hema tocrit & $51.4+/-9.2 *$ & $46.9+-7.5$ \\
\hline Creatinine & $58.8+1-15.4$ & $66+t-16.8^{*}$ \\
\hline
\end{tabular}

tp $<0.05$ term versus preterm group.

\section{PO-0296 PREVALENCE OF INTERCURRENT ILLNESS IN CHILDREN AGED 0-4 YRS, WHO PRESENT TO PAEDIATRIC A\&E WITH MINOR HEAD INJURY, AND THEIR MANAGEMENT WITH REGARDS IMAGING}

C Mckenna, C Stewart, W Teranaka, E Abrahamson. Paediatric Emergency Department, Chelsea and Westminster Hospital, London, UK

10.1136/archdischild-2014-307384.947

Background Minor head injury is a common presentation to Paediatric Emergency Departments (PED). Various clinical decision rules exist to help clinicians decide which patients require radiological investigation, several include 3 or more discreet vomits. Anecdotally there is a subgroup of patients with minor head injury (MHI) who have intercurrent illnesses (ICI), however there is a paucity of research into this group and their management, particularly with regards imaging.

Objectives

1) To quantify the proportion of children (aged $0-4$ yrs) who present to the PED with MHI and have symptoms of ICI.

2) To determine if these children are more likely to vomit than their counterparts.

3) To establish current clinical practice for children with ICI and vomiting post $\mathrm{MHI}$.

Results Of 1203 children aged $0-4$ yrs presenting to a PED in central London between April 2011 and 2012 with minor head injury, $88(7.3 \%)$ had symptoms of an ICI. Children who had symptoms of ICI $(38 / 88)$ were more likely to vomit than those who did not $(92 / 1112)(\mathrm{p}<0.001)$. Of 16 patients who had an ICI and 3 or more vomits following head injury only $2 / 16$ (12.5\%) underwent CT head compared with 8/27 (29.6\%) in the non ICI group. There were no cases of brain injury in either group.

Conclusion Minor head injury occurs frequently in the presence of ICI in younger children. These patients are more likely to vomit and clinicians are consciously contradicting current guidelines and interpreting imaging criteria within a clinical context.

\section{PO-0297 CHILDREN PRESENTING TO THE EMERGENCY DEPARTMENT WITH HEADACHE AS PRIMARY COMPLAINT ARE NOT LIKELY TO HAVE A BRAIN TUMOUR}

G van Berlaer, K Mortelmans, I Hubloue. Department of Emergency and Disaster Medicine, UZ Brussel, Brussels, Belgium

10.1136/archdischild-2014-307384.948
Background Headache is a very common complaint in children presentating to the Paediatric Emergency Department (PED) and causes concern in parents and physicians regarding malignant intracranial processes. Aim of this study is to search for malignant etiologies in children presenting to the PED with headache as primary complaint.

Patients and methods All digital medical charts of children $<16$ years presenting to the PED between August 2011 and August 2013 with headache as primary complaint were retrospectively reviewed. Children with history of intracranial tumorsor surgery, recurrent headache under investigation or treatment, or traumatic headache were excluded. Age, gender, brain imaging and final diagnoses were registered.

Results Of all 34,336 children seen at the PED during 2 years, $117(0.3 \%)$ consulted with headache as primary complaint: 61 boys (8.4 years \pm 3.4 ) and 56 girls (8.6 years \pm 3.7 ). Brain imaging (CT or MRI) was performed in 33/117 children (28\%) because of clinical suspicion of intracranial processes: presence of associated neurological symptoms, valsalva manoeuvre increasing pain intensity, evolution with intensifying pain, recurrent focal pain, change in pattern/intensity, lack of response to analgesics and aberrant neurological features. Most frequent medical conclusions were "headache related to a viral infection", "migraine", "tension type headache", "psychosomatic headache" and "headache without identified origin". None of these patients suffered from a brain tumour.

Conclusions Children presenting to the PED with headache as primary complaint are not likely to suffer from a malignant brain tumour. More frequent their headache is related to a concurrent viral infection.

\section{PO-0298 LIMITING WORKING SHIFT TIMES FOR PAEDIATRIC EMERGENCY DEPARTMENT PHYSICIANS REDUCES NUMBER OF COMPLAINTS BY PATIENTS' PARENTS}

G van Berlaer, V Van Mulders, I Hubloue. Department of Emergency and Disaster Medicine, UZ Brussel, Brussels, Belgium

\subsection{6/archdischild-2014-307384.949}

Background Paediatric trainees have always worked many and long shifts during their education, especially while providing 24/ 7 continuity of care at theDepartment of Emergency Medicine. It is depicted that longer shifts lead to more medical errors and less patience with colleagues and patients.

This study aims to quantify the effect of reducing working shift duration for paediatric trainees on the number of complaints by parents of paediatric patients.

Methods The study is conducted in the Paediatric Emergency Department (PED) ofa large tertiary hospital. Paediatric residents used to work shifts of over 24 h before, but work was reorganised introducing a rotational working scheme in 2008 reducing shifts to an absolute maximum of $14 \mathrm{~h}$. All complaints filed by parents - either through the PED mailbox or via the Complaints Mediation Service of the hospital - were analysed. Periods before and after introduction of the rotational reduced shift system were compared regarding number of complaints, severity of these complaints, and number of reported verbal or physical aggressions.

Results Between2003 and 2012, more than 150,000 patients presented to the PED. Less than $1 \%$ of these contacts led to a complaint. The number of complaints filed by parents still dropped significantly after reducing working shift times for 